\section{Measurement of 50-fs (rms) Electron Pulses}

Hung-chi Lihn, Pamela Kung, Helmut Wiedemann Applied Physics Department and

Stanford Linear Accelerator Center. Stanford University, Stanford, California 94309, USA

\section{David Bocek}

Physics Department and

Stanford Linear Accelerator Center, Stanford University, Stanford, California 94309, USA

\section{Abstract}

Electron pulses generated at the Stanford SUNSHINE facility with $(2-4.6) \times 10^{8}$ electrons per microbunch have been measured as short as $50 \mathrm{fs}(\mathrm{rms})$. The bunch length is determined by optical autocorrelation via a far-infrared Michelson interferometer using coherent transition radiation emitted at wavelengths longer than and equal to the bunch length. This frequency-resolved autocorrelation method demonstrates a better sub-picosecond resolving power than any existing time-resolved method. The experimental setup and the results will be described.

\section{INTRODUCTION}

In the development of particle accelerators, the measurement of the electron bunch length in the longitudinal phase space has played a very important role. Through bunch length measurements, it is possible to examine different bunch generation and compression techniques and, hence, find not only ways of optimizing accelerators' performance but also guidance to the design of future linear colliders, free-electron lasers, and high-intensity coherent light sources in the far-infrared regime.

To measure the bunch length, two approaches can be taken: the timeresolved and the frequency-resolved technique. The time-resolved technique uses fast electronics to resolve beam-generated light pulses in the time domain and derives the longitudinal bunch distribution. When the bunch length is reduced through the development of accelerators, faster processing speed and more advanced technology are required to achieve higher resolution. As a result, the highest time resolution achieved by the fastest time-resolved device, the streak camera, is on the order of a picosecond. However, this resolving power is not enough for facilities like SUNSHINE at Stanford University $(1,2)$, where electron bunches can be compressed to the 100-fs range. To resolve such short electron bunches, new bunch length measuring technique must be used.

The frequency-resolved technique, on the other hand, resolves the frequency content of beam-generated light pulses in the frequency domain and deduces the particle distribution from the frequency information. Unlike time-resolved methods, this technique does not require fast processing speed, and the necessary broad bandwidth for short pulses can be achieved by optical methods.

Contributed to the 6th Beam Instrumentation Workshop (BIW 94), Vancouver, B.C., Canada, October 2-6, 1994
Based on this technique, an optical autocorrelation method, similar to that used to characterize femtosecond laser pulses(3), has been proposed for subpicosecond bunch length measurement(4). It utilizes the coherence property of the radiation emitted by short electron bunches to generate the optical autocorrelation via a far-infrared Michelson interferometer. By analyzing this autocorrelation measurement, it is possible to determine the electron bunch length down to sub-picosecond resolution.

In this paper, we will describe the experiment using this new autocorrelation method to measure the sub-picosecond electron bunches generated at SUNSHINE. We first describe the principle of bunch compression used at this facility and then discuss the experimental setup and the results.

\section{CONCEPTUAL BACKGROUND}

The bunch generation and compression system used at SUNSHINE, as

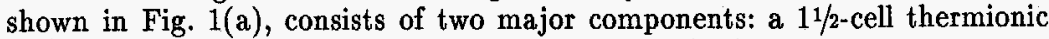
RF gun and an alpha magnet with energy filters $(1,2)$. The RF gun operating at $2856 \mathrm{MHz}$ produces $2.5-\mathrm{MeV}$ electron bunches in which the electrons are distributed along a thin line in the energy-time phase space with higher energy electrons located at earlier time and lower energy electrons, later time. These energy-time correlated bunches are then steered into the alpha magnet for compression. The magnet will guide the electrons in the magnet along $\alpha$-shaped paths with higher energy electrons following longer paths and vice versa; hence, the earlier electrons in the bunch, which have higher energy, will spend more time in the magnet by following longer paths while the later electrons, less time. By correctly setting the magnet's strength, it is possible to compress part of the electron bunch into sub-picosecond duration. This optimally compressed part is then selected by energy filters and transported through a $30-\mathrm{MeV}$ linear accelerator and a beam transport line to the radiation source point. When transporting the electron bunch, the velocity spread in the bunch can cause significant bunch lengthening; therefore, it is necessary to compensate for this effect by overcompressing the bunch so that the minimum bunch length is reached at the source point.

When these short electron bunches emit radiation, the total radiation is incoherent at wavelengths shorter than the bunch length and temporally coherent at wavelengths longer than and equal to the bunch length(5). The intensity of the coherent part exceeds that of the incoherent part by a factor of $N_{e}$ the number of electrons in each bunch. This coherent intensity enhancement makes it easier to detect the radiation from sub-picosecond electron bunches in the far-infrared regime. The radiation spectrum is the Fourier transform of the longitudinal bunch distribution; hence, the determination of the bunch length will become possible once this spectrum is measured by the frequency-resolved technique. 


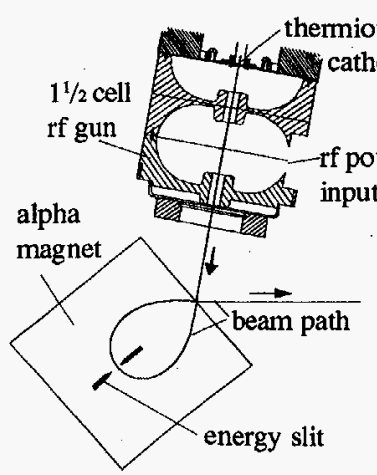

(a) Bunch generation and compression system

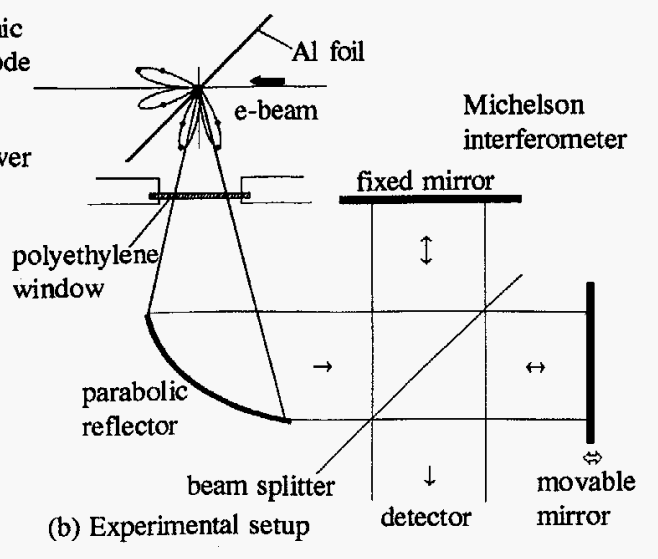

Figure 1. Schematic diagrams of (a)the bunch generation and compression system used at SUNSHINE and (b)the experimental setup.

\section{EXPERIMENTAL SETUP}

As stated in the previous section, 2.5-MeV electron bunches are produced by the RF gun every $350 \mathrm{ps}$ for a macropulse duration of about $1 \mu \mathrm{s}$ at a repetition rate of $10 \mathrm{~Hz}$. These electron bunches, with a macropulse current of $700-900 \mathrm{~mA}$ at the gun exit, are guided into the alpha magnet. The magnet compresses part of each bunch into sub-picosecond duration and the undesirable part of the beam is filtered out. Finally, these sub-picosecond electron bunches, with $(2-4.6) \times 10^{8}$ electrons each, are accelerated to $30 \mathrm{MeV}$ and transported to the radiation source point.

At the radiation source point, as shown in Fig. 1(b), transition radiation is generated when the electrons pass through a 25.4- $\mu \mathrm{m}$-thick Al foil. The foil supported by a copper ring is oriented at a $45^{\circ}$ angle to the beam direction so that the backward transition radiation is emitted in the direction normal to the beam path and can easily be extracted from the vacuum chamber through a 1-mm-thick high-density polyethylene window of $19 \mathrm{~mm}$ diameter. Since the backward transition radiation is emitted at the Al surface, the focal point of an off-axis paraboloidal mirror is aligned with this surface to make the divergent radiation parallel without introducing optical path difference to the extracted light pulse. The parallel light then enters a far-infrared Michelson interferometer.

The interferometer consists of a 12.7- $\mu \mathrm{m}$-thick Mylar beamsplitter, a fixed and a movable first surface mirror, and a room-temperature bolometer. The movable mirror is controlled by a Newport $850-\mathrm{B} 25-\mathrm{mm}$ actuator through a Newport PMC200-P 2-axis controller which in turn is controlled by a 486-based
$\mathrm{PC}$ via an RS-232 port. The bolometer consists of a Molectron P1-65 $\mathrm{LiTaO}_{3}$ pyroelectric detector of $5 \mathrm{~mm}$ diameter and a pre-amplifier. This bolometer is designed to measure the integrated radiation energy from each 1- $\mu$ s macropulse with a responsivity of pre-amplification $\times 1.21 \times 10^{3} \mathrm{~V} / \mathrm{J}$ and is attached to a copper light cone, which will collect the parallel light into the bolometer. The bolometer signal is digitized into the computer through a National Instruments AT-MIO-16F-5 data acquisition board. With these computer interfaces, the autocorrelation measurement can be performed automatically through the program under the LabVIEW control environment implemented on the computer.

\section{RESULTS AND DISCUSSION}

By measuring the bolometer signal as a function of the position of the movable mirror through the computer program, typical 16 -mm-long autocorrelation scans with $10-\mu \mathrm{m}$ mirror step size were performed with a good signalto-noise ratio. For example, the bolometer background noise was measured around $2 \mathrm{mV}$ while the bolometer signal was measured about $200 \mathrm{mV}$ after pre-amplification. However, in all the presented data, the pre-amplification was removed.

For an ideal beamsplitter which equally splits the intensity of the incoming radiation into two mirror arms, the intensity of the radiation combined at the detector, which will produce a proportional bolometer signal, can be expressed in the time domain as

$$
\begin{aligned}
I(\delta) & =\int_{-\infty}^{+\infty}\left|R T\left\{E(t)+E\left(t+\frac{\delta}{c}\right)\right\}\right|^{2} d t \\
& =2|R T|^{2}\left\{\operatorname{Re} \int_{-\infty}^{+\infty} E(t) E^{*}\left(t+\frac{\delta}{c}\right) d t+\int_{-\infty}^{+\infty}|E(t)|^{2} d t\right\}
\end{aligned}
$$

or in the frequency domain as

$$
\begin{aligned}
I(\delta) & =\int_{-\infty}^{+\infty}\left|R T E(\omega)\left(1+e^{i \omega \frac{\delta}{c}}\right)\right|^{2} d \omega \\
& =2\left\{\operatorname{Re} \int_{-\infty}^{+\infty}|R T|^{2}|E(\omega)|^{2} e^{i \omega \frac{\delta}{c}} d \omega+\int_{-\infty}^{+\infty}|R T|^{2}|E(\omega)|^{2} d \omega\right\}
\end{aligned}
$$

where $\delta$ is the optical path difference between both mirror arms, and $R$ and $T$ are the amplitude reflection and transmission coefficients with $|R|^{2}=|T|^{2}=$ 1/2. Equation 1 and 2 are related by the Fourier transform of $E(t)$. Furthermore, the interferogram is defined as $I(\delta)-I_{\infty}$, where $I_{\infty}$ is $I(\delta \rightarrow \pm \infty)=$ $2|R T|^{2} \int_{-\infty}^{+\infty}|E(t)|^{2} d t=2 \int_{-\infty}^{+\infty}|R T|^{2}|E(\omega)|^{2} d \omega$. The interferogram is, indeed, the autocorrelation of the light pulse (see Eq. 1), and its Fourier transform is the power spectrum of the pulse (see Eq. 2). $I(\delta)$ is equal to $I_{\infty}$ for $|\delta|$ much larger than the pulse length and rises to the maximum of $2 I_{\infty}$ at $\delta=0$. Hence, the width of this peak around $\delta=0$ in the interferogram can be used 


\section{DISCLAIMER}

Portions of this document may be illegible in electronic image products. Images are produced from the best available original document. 


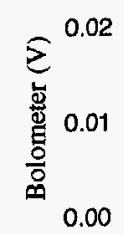

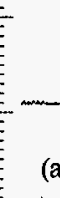

(a) Autocorrelation scan

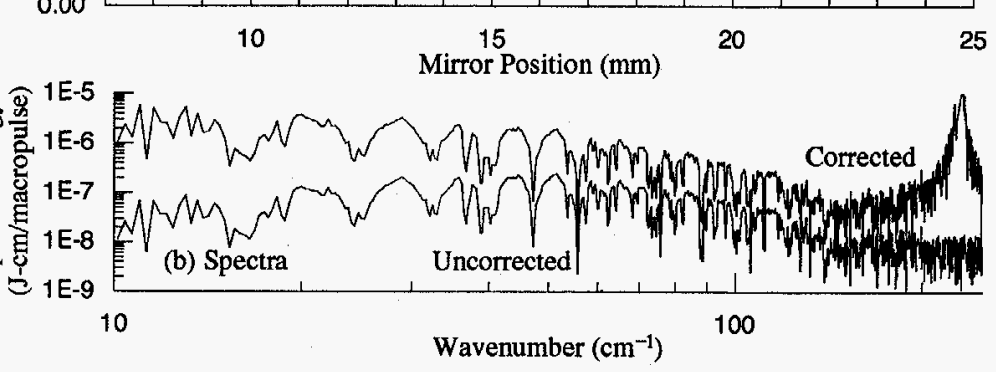

Figure 2. Typical 16-mm-long autocorrelation scan and derived spectra for radiation from sub-picosecond electron pulses. The autocorrelation scan is shown in (a). The raw spectrum and the one corrected for the Mylar beamsplitter efficiency are shown in (b).

to estimate the pulse length (which is equivalent to the bunch length). For example, the full width at half maximum (FWHM) of the interferogram is equal to the bunch length for a rectangular bunch distribution and is equal to $4 \sqrt{\ln 2} \sigma_{z}$ for a Gaussian bunch distribution with an equivalent bunch length of $\sqrt{2 \pi} \sigma_{z}(=0.75 \mathrm{FWHM})$. Most real bunch distributions are neither rectangular nor Gaussian, and the equivalent bunch length can be estimated as $75 \%$ to $100 \%$ of the FWHM of the interferogram. A typical autocorrelation measurement, as shown in Fig. 2(a), exhibits the sharp rise in signal to about twice of $I_{\infty}$ when pulses from both mirror arms overlap.

However, a suitable beamsplitter used for the far-infrared regime does not provide constant $1 / 2: 1 / 2$ reflection and transmission at all frequencies but those varying as functions of frequency. This departure from the ideal beamsplitter is caused by the interference of light reflected from both surfaces of the Mylar beamsplitter, which is equivalent to thin film interference. Because of this interference, the efficiency of the 12.7- $\mu$ m-thick Mylar beamsplitter, which is defined as $|R T|^{2}$, increases from zero at zero frequency to the maximum value of 0.17 at $115 \mathrm{~cm}^{-1}$ and then drops to zero again at $230 \mathrm{~cm}^{-1}$, the first singularity of the beamsplitter, where light reflected from both surfaces of the beamsplitter forms destructive interference. Hence, the beamsplitter thickness of $12.7 \mu \mathrm{m}$ is specially chosen to include the whole expected spectrum within the frequency range up to its first singularity.

The raw radiation spectrum of the autocorrelation measurement and the

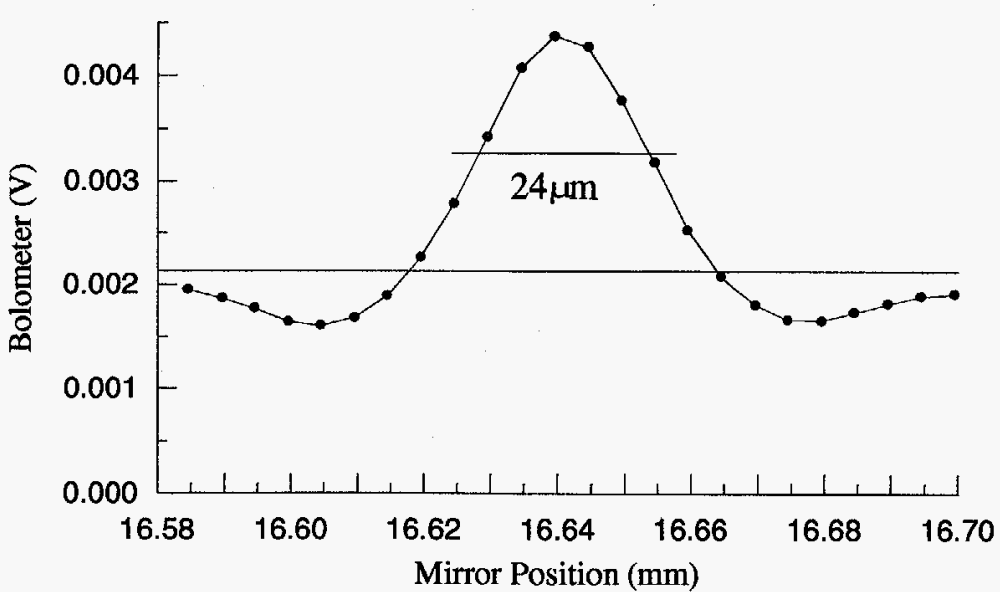

Figure 3. Fine autocorrelation scan of the main peak for the shortest electron bunch length obtained at SUNSHINE. The measurements are shown as $\bullet$ with mirror step size of $5 \mu \mathrm{m}$.

one corrected for the Mylar beamsplitter efficiency are shown in Fig. 2(b). The total 16-mm mirror movement corresponding to $32-\mathrm{mm}$ optical path difference results in a spectral resolution of $0.3125 \mathrm{~cm}^{-1}$. Below $10 \mathrm{~cm}^{-1}$, the spectra are believed to be contaminated by slow drift of machine parameters during the half-hour measurement. The whole raw spectrum is well contained within the frequency range up to the first singularity of the beamsplitter. The spike around the first singularity of the beamsplitter in the corrected spectrum indicates a large over-correction due to the poor signal-to-noise ratio in this spectral range. The roll-off rate of the corrected spectrum at high frequency is closer to that of a rectangular distribution rather than that of a Gaussian distribution. The multitude of absorption lines in the spectrum are identified as water absorption lines(6) caused by the humidity in ambient air.

Utilizing the bunch information from the autocorrelation measurement, the performance of the accelerator was optimized to produce the shortest bunch length. With this optimization effort, the shortest bunch length was obtained and shown in Fig. 3. The FWHM of the peak in the interferogram is $24 \mu \mathrm{m}$ of mirror movement which is equivalent to $48 \mu \mathrm{m}$ of optical path difference. Hence, the equivalent bunch length is estimated as $36 \mu \mathrm{m}$ (Gaussian) to $48 \mu \mathrm{m}$ (rectangular) or, in the time scale, as $120 \mathrm{fs}$ (Gaussian) to $160 \mathrm{fs}$ (rectangular). The rms bunch length is calculated as $46 \mathrm{fs}$ (rectangular) to $48 \mathrm{fs}$ (Gaussian). 


\section{CONCLUSION}

In summary, we have shown the results of using an optical autocorrelation method to measure the length of sub-picosecond electron bunches generated at SUNSHINE. The rms bunch length is measured about $50 \mathrm{fs}$. This new method has demonstrated a very good sub-picosecond resolving power which out-performs any existing time-resolved method; hence, it may be applied to any short bunch length measurement task. Furthermore, the Michelson interferometer used in this method fits on a $20 \times 30 \mathrm{~cm}^{2}$ breadboard and is easily transportable. Combining this compact interferometer with the easily manageable transition radiation and the room-temperature bolometer, this new device would become a convenient diagnostic instrument for accelerators.

\section{ACKNOWLEDGEMENT}

The authors would like to thank for the technical support by M. Baltay, G. Husak, J. Sebek, R. Theobald, and J. Weaver in this experiment. We also appreciate the useful introduction on the techniques of far-infrared experimentation received from $P$. Richards and the help to use some critical equip ment provided by A. Schwettman and T. Smith. This work was supported by the Department of Energy, Office of Basic Energy Sciences (contract No. DE-AC03-76SF00515) and the Office of Naval Research (contract No. N0001491-C-0170).

\section{REFERENCES}

1. Kung, P. H., Lihn, H.-C., Bocek, D., and Wiedemann, H., "High-intensity coherent FIR radiation from sub-picosecond electron bunches," in Gas, Metal Vapor, and Free-Electron Lasers and Applications, edited by Similey, V. N. and Tittel, F. K., Proc. SPIE 2118, 191(1994).

2. Kung, P. H., Lihn, H.-C., Bocek, D., and Wiedemann, H., "Generation and measurement of 50 -fs (rms) electron pulses," Phys. Rev. Lett. 73 , 967(1994).

3. Fork, R.L., Greene, B. I., and Shank, C. V., "Generation of optical pulses shorter than $0.1 \mathrm{psec}$ by colliding pulse mode locking," Appl. Phys. Lett. 38, 671(1981).

4. Barry, W., "An autocorrelation technique for measuring sub-picosecond bunch length using coherent transition radiation," in Proceedings of the Workshop on Advanced Beam Instrumentation 1, KEK, Tsukuba, Japan, April 22-24, 1991 (unpublished).

5. Nodvick, J. S. and Saxon, D. S., "Suppression of coherent radiation by electrons in a synchrotron," Phys. Rev. 96, 180(1954).

6. Richards, P. L., "High-resolution Fourier transform spectroscopy in the far-infrared," J. Opt. Soc. Am. 54, 1474(1964).

\section{DISCLAIMER}

This report was prepared as an account of work sponsored by an agency of the United States Government. Neither the United States Government nor any agency thereof, nor any of their employees, makes any warranty, express or implied, or assumes any legal liability or responsibility for the accuracy, completeness, or usefulness of any information, apparatus, product, or process disclosed, or represents that its use would not infringe privately owned rights. Reference herein to any specific commercial product, process, or service by trade name, trademark, manufacturer, or otherwise does not necessarily constitute or imply its endorsement, recommendation, or favoring by the United States Government or any agency thereof. The views and opinions of authors expressed herein do not necessarily state or reflect those of the United States Government or any agency thereof. 